\title{
Recidiva de osteosarcoma: detección por gammagrafía y SPECT/CT empleando ${ }^{99}$ Tc-sestamibi
}

\author{
Eiber Alexis Briones-Velázquez, ${ }^{\ddagger}, *$ Cristian Luna-de-la-Luz ${ }^{\S}$ \\ ${ }^{\ddagger}$ Residente de Medicina Nuclear e Imagenología Molecular; ${ }^{\S}$ Servicio de Medicina Nuclear. Hospital de Especialidades \\ Antonio Fraga Mouret, Centro Médico Nacional "La Raza”, Instituto Mexicano del Seguro Social, Ciudad de México.
}

\begin{abstract}
RESUMEN
Presentamos las imágenes de gammagrafía de una paciente de 17 años de edad con sospecha de recidiva de osteosarcoma en el tercio distal de la tibia derecha después de cinco años de estar en vigilancia. Para confirmar la recurrencia, se decidió efectuar una gammagrafía y posteriormente un SPECT/CT empleando el radiofármaco $\mathrm{Tc}^{99} \mathrm{~m}$-sestamibi. En las imágenes se evidenció incremento anormal de actividad metabólica a nivel de la meseta tibial derecha sugiriendo actividad tumoral, dichos hallazgos se corroboraron mediante histopatología, la cual confirmó el diagnóstico de osteosarcoma convencional.
\end{abstract}

Palabras clave: Osteosarcoma, gammagrafía, SPECT/CT, $\mathrm{Tc}^{99} \mathrm{~m}$-sestamibi.

\section{INTRODUCCIÓN}

El osteosarcoma es el tumor óseo de origen primario más frecuente en Pediatría, el cual muestra una incidencia mundial de 3.4 casos por millón de personas al año. En México para el año 2000 representó $4.5 \%$ del total de las neoplasias en niños, que corresponde a $74 \%$ de los tumores óseos, ocupando el séptimo lugar como causa de mortalidad por cáncer en Pediatría. ${ }^{1,2}$

\footnotetext{
* Correspondencia: EABV, eabv07@hotmail.com Conflicto de intereses: Los autores declaran que no tienen. Citar como: Briones-Velázquez EA, Luna-de-la-Luz C. Recidiva de osteosarcoma: detección por gammagrafía y SPECT/CT empleando ${ }^{99 m}$ Tc-sestamibi. Rev Mex Pediatr. 2020; 87(1):38-40. doi: $10.35366 / 93267$

[Osteosarcoma recurrence: detection by scintigraphy and SPECT/ CT using ${ }^{99 m}$ Tc-sestamibi]
}

\begin{abstract}
We present scintigraphic images of a 17-year-old female patient with suspected osteosarcoma recurrence in the distal third of the right tibia, after five years of being under surveillance. To confirm recurrence, it was decided to carry out a scintigraphy and later a SPECT / CT using the Tc99msestamibi radiopharmaceutical. Images showed abnormal increase metabolic activity at the level of the right tibial plateau suggesting tumor activity. Histopathology confirmed the diagnosis of osteosarcoma.
\end{abstract}

Keywords: Osteosarcoma, scintigraphy, SPECT/CT, Tc ${ }^{99} \mathrm{~m}-$ sestamibi.

Esta neoplasia se presenta más comúnmente en la segunda década de la vida entre los 10 y 15 años de edad. Aunque puede afectar cualquier hueso, tiene predilección por las metáfisis de los huesos largos, siendo los más frecuentes el fémur distal y la tibia proximal. Los datos clínicos principales son dolor, aumento de tamaño y disminución de la movilidad de la región afectada; además de pérdida de peso y malestar generalizado. ${ }^{2,3}$

En lo referente al diagnóstico por imagen, existen diversas modalidades que ayudan a la sospecha de la enfermedad al inicio del padecimiento para evaluar la evolución y recidivas. Éstas incluyen la radiología convencional, resonancia magnética, tomografía computarizada (TC), tomografía por emisión de positrones (TEP), tomografía por emisión de fotón único (SPECT) y a la gammagrafía ósea (GO). En la GO se utilizan difosfonatos marcados con tecnecio-99 metaestable ( ${ }^{99 \mathrm{~m}} \mathrm{Tc}$ ), que pueden ser metilendifosfonato $\left({ }^{99 \mathrm{~m}} \mathrm{Tc}-\mathrm{MDP}\right) \mathrm{o}$ 
hidroximetilen difosfonato ( $\left.{ }^{99 \mathrm{~m}} \mathrm{Tc}-\mathrm{HMDP}\right)$. Estos radiofármacos se unen a los cristales de hidroxiapatita en la matriz mineral del hueso, principalmente en sitios de recambio osteoblástico. En tumores óseos la sensibilidad es de $62-100 \%$ y la especificidad de $78-100 \%$ con estos dos radiofármacos; sin embargo, más recientemente se ha propuesto el uso de ${ }^{99 \mathrm{~m}} \mathrm{Tc}$-sestamibi. ${ }^{4-6}$

Por otro lado, el uso de sistemas híbridos como el SPECT/TC permite mejorar el diagnóstico, ya que el tejido que capta el radiofármaco se observa con mayor definición, así como la localización anatómica de la masa tumoral dentro de un órgano al excluir la presencia del compuesto en vasos, sistema urinario y tracto gastrointestinal, lo cual mejora la especificidad en el diagnóstico. ${ }^{7}$

El objetivo de este trabajo es mostrar la utilidad de la gammagrafía y su posterior complementación con SPECT/CT utilizando el radiofármaco ${ }^{99 \mathrm{~m}} \mathrm{Tc}$-sestamibi para el diagnóstico de la recurrencia tumoral en una paciente con osteosarcoma.

$\mathrm{Al}$ momento del estudio, la paciente tenía 17 años de edad, con diagnóstico de osteosarcoma a los 12 años, localizado en el tercio distal de la tibia derecha. Se encontraba en vigilancia después de terminar tratamiento médico y quirúrgico, siendo necesario efectuar amputación transtibial de la extremidad inferior derecha. Fue referida al Servicio de Medicina Nuclear para realizar un GO con ${ }^{99 \mathrm{~m}} \mathrm{Tc}-\mathrm{HMDP}$ por dolor de un mes de evolución en el muñón, el cual no estaba relacionado a trauma o al uso de la prótesis. Al momento de su valoración no había aumento del volumen de la región, limitación al movimiento del muñón o algún dato de inflamación.

Tomando en cuenta el historial médico previo de la paciente, se decidió no utilizar el radiofármaco ${ }^{99 \mathrm{~m} T c-}$ HMDP, debido a que aunque la GO con difosfonatos tiene alta sensibilidad, su especificidad es baja a consecuencia de un gran número de factores (cambios degenerativos, cambios óseos postquirúrgicos, artritis, estrés o fracturas óseas, uso de prótesis, neoplasias, trauma u osteomielitis) que pueden incrementar la actividad osteoblástica y por consiguiente, aumentar la captación de los difosfonatos afectando la interpretación del estudio, aunado a que no pueden diferenciar entre tumores benignos y malignos. Por lo anterior, y ante la sospecha de recidiva tumoral, se decidió emplear ${ }^{99 \mathrm{~m}} \mathrm{Tc}$-sestamibi, ya que este radiofármaco se incorpora al tejido metabólicamente activo y es retenido en el interior de las mitocondrias, gracias a la carga negativa de la membrana interna y de la matriz mitocondrial. Este radiofármaco es un catión lipofílico, lo que permite su ingreso a través de las membranas celulares y ser retenido debido a la diferencia de sus cargas electroestáticas. En el caso de la célula neoplásica el incremento del potencial negativo transmembrana secundario al aumento de los requerimientos metabólicos promueve la captación de este radiofármaco en el interior de estas células. ${ }^{5,8}$

Para realizar la gammagrafía inicialmente se inyectaron $20 \mathrm{mCi}$ de ${ }^{99 \mathrm{~m} T c-s e s t a m i b i . ~ D e s p u e ́ s ~ d e ~} 120 \mathrm{minu}-$ tos se adquirió el rastreo corporal total en proyecciones anterior y posterior en un equipo Siemens E-cam doble cabezal bajo las siguientes condiciones: velocidad de escaneo $10 \mathrm{~cm} /$ minuto, matriz $256 \times 1024$ pixeles, zoom: 1, colimador de baja energía y alta resolución (LEHR), fotópico centrado a $140 \pm 20 \% \mathrm{keV}$. A continuación se procedió a realizar un SPECT/CT en un equipo Philips Precedence doble cabezal de 16 cortes. El equipo permite la fusión de imágenes con reconstrucción en los planos sagitales, coronales y axiales. Las condiciones de trabajo fueron las siguientes: órbita circular con arco de $360^{\circ}, 64$ proyecciones en una matriz de $64 \times$ 64 , tiempo por proyección de 20 segundos, fotópico
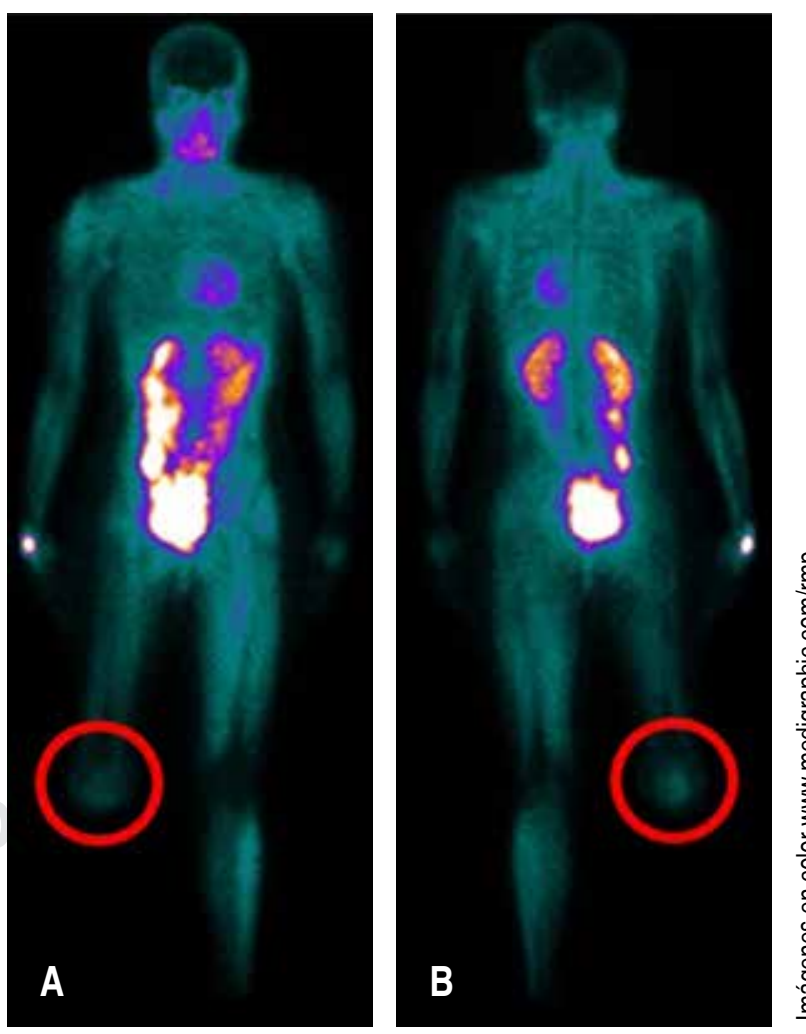

Figura 1: Rastreo corporal total con ${ }^{99 \mathrm{mT}} \mathrm{C}$-sestamibi en proyección anterior A) y posterior B). 

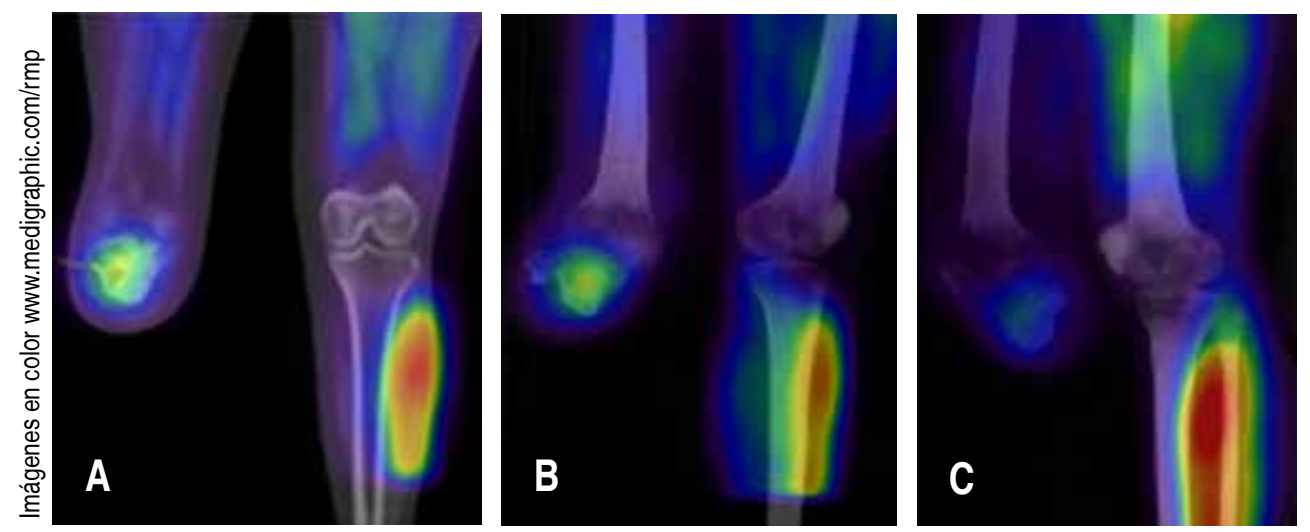

\section{Figura 2:}

Imágenes obtenidas por fusión y reconstrucción SPECT/CT:

A) Proyección anterior.

B) Proyección oblicua anterior derecha.

C) Proyección oblicua anterior izquierda.

centrado a $140 \pm 20 \% \mathrm{keV}$, colimador LEHR, filtros de procesamiento: Butterworth, TC: matriz $512 \times 512,130$ $\mathrm{kV}, 140 \mathrm{mAs}$, pitch 1.

En el rastreo corporal total se observó la biodistribución (glándulas salivales, tiroides, miocardio, músculos esqueléticos) y la eliminación (hepatobiliar, intestinal, renal) habituales del radiofármaco. En las áreas señaladas con círculos rojos se aprecia una zona de captación anormal del radiofármaco en el muñón, lo cual no es evidente en la región homóloga contralateral (Figura 1).

En la imagen por SPECT/CT de miembros inferiores se observa la biodistribución esperada del radiofármaco (musculosesqueléticos). La valoración de todas las imágenes indican claramente (Figura 2) el aumento de la actividad metabólica en la meseta tibial derecha sugiriendo actividad tumoral, dichos hallazgos se corroboraron mediante el estudio histopatológico, el cual confirmó el diagnóstico de osteosarcoma convencional.

Por lo expuesto, consideramos que el uso de la gammagrafía complementado con SPECT/TC utilizando el radiofármaco ${ }^{99 \mathrm{~m}} \mathrm{Tc}$-sestamibi es una opción adecuada para determinar la viabilidad tumoral en pacientes con sospecha inicial de osteosarcoma o de recidiva tumoral.

\section{REFERENCIAS}

1. Misaghi A, Goldin A, Awad M, Kulidjian A. Osteosarcoma: a comprehensive review. SICOT-J. 2018; 4: 1-8.

2. Diagnóstico oportuno de osteosarcoma en niños y adolescentes en primer y segundo nivel de atención médica. México: Secretaría de Salud; 2013.

3. Durfee R, Mohammed M, Luu H. Review of osteosarcoma and current management. Rheumatol Ther. 2016; 3(2): 221-243.

4. American Cancer Society. Bone Cancer Early Detection, Diagnosis, and Staging. 2019. www.cancer.org/content/dam/CRC/ PDF/Public/8770.00.pdf.

5. Ziessman H, O'Malley J, Thrall J. Nuclear medicine: the requisites. $4^{\text {th }}$ ed. Philadelphia, USA: Elsevier/Saunders; 2014.

6. Jabeen A, Raza H, Ahmed B, Minhaj M, Mushtaq S, Khan L et all. 99mTc-MIBI Scintigraphy to differentiate malignancies from benign lesions detected on planar bone scans. Egypt J Radiol Nucl Med. 2016; 47: 267-273.

7. Perera-Pintado A, Torres-Aroche LA, Vergara Gil A, Batista Cuéllar JF, Prats Capote A. SPECT/CT: principales aplicaciones en la medicina nuclear. Nucleus. 2017; 62: 2-9.

8. Urbano N, Scimeca M, Bonanno E, Schillaci O. 99mTc sestamibi SPECT: a possible tool for early detection of breast cancer lesions with high bone metastatic potential. Future Oncol. 2019; 15(5): 455-457. 